\title{
ON A COHOMOLOGY THEORY FOR PAIRS OF GROUPS
}

\author{
LUIS RIBES
}

Let $H$ be a subgroup of a group $G$, and let $A$ be a left $G$-module. Consider the abelian group

$$
X(G, H, A)=\left\{f: G \rightarrow A \mid f(x y)=x f(y)+f(x), f_{\mid H}=0\right\}
$$

of crossed homomorphisms from $G$ to $A$ vanishing on $H$. Clearly this is a left-exact functor in the category ${ }_{G} \mathbb{N}$ of left $G$-modules. The $n$th right derived functor of $X(G, H,-)$ in ${ }_{G} \Re$ is denoted by $H^{n+1}(G, H,-)$. The group $H^{n}(G, H, A), A \in{ }_{G} \Re$, is called the $n$th cohomology group of the pair $(G, H)$ with coefficients in $A$. These groups were first described and studied by M. Auslander in [1], who also found the sequence of Proposition 1.2.

In this note we prove an excision property for the functors $H^{n}(G, H,-)$, Theorem 2.2, and we find a direct sum decomposition of them under suitable conditions, Propositions 2.3 and 2.5. From this one deduces by standard methods a Mayer-Vietoris type sequence for the cohomology of groups, Proposition 2.6.

The results in this paper are part of the author's doctoral dissertation at the University of Rochester. The author wishes to thank C. E. Watts for his advise and encouragement.

1. Let $H \subset G$ be groups, and let $A \in{ }_{G} \mathfrak{M}$. Then $A^{*}=\operatorname{Hom}_{H}(Z G, A)$ is a left $G$-module in the obvious way ( $Z G$ denotes the integral group ring, and $\left.\operatorname{Hom}_{H}(-,-) \equiv \operatorname{Hom}_{Z H}(-,-)\right)$. Let $Z$ be the group of integers with $G$-structure defined by $x n=n, x \in G, n \in Z$. Then one has the natural isomorphisms

$$
\begin{aligned}
\operatorname{Hom}_{G}\left(Z, A^{*}\right) & =\operatorname{Hom}_{G}\left(Z, \operatorname{Hom}_{H}(Z G, A)\right) \\
& \approx \operatorname{Hom}_{H}\left(Z G \otimes_{G} Z, A\right) \approx \operatorname{Hom}_{H}(Z, A) .
\end{aligned}
$$

Hence, since every $G$-injective module is $H$-injective, cf. [4, p. 31 , Proposition 6.2a], one has

$$
H^{n}\left(G, A^{*}\right) \approx H^{n}(H, A)
$$

for $A \in{ }_{G} \Re$. ( $A^{*}$ is exact in ${ }_{G} \Re($ and preserves injectives.)

Now let $\gamma: A \rightarrow A^{*}$ be the $G$-monomorphism defined by $\gamma(a) x=x a$, $a \in A, x \in G$. Let $\Gamma=\operatorname{coker} \gamma$.

Lemma 1.1. $\operatorname{Hom}_{G}(Z, \Gamma) \approx X(G, H, A)$ as functors in ${ }_{G} \Re$.

Proof. Notice that $\Gamma=A^{*} / \operatorname{Im} \gamma$. We make the identifications

Received by the editors May 16, 1968. 


$$
\begin{aligned}
A^{*} & =\operatorname{Hom}_{H}(Z G, A)=\{f: G \rightarrow A \mid f(x y)=x f(y), x \in H, y \in G\}, \\
\operatorname{Im} \gamma & =\operatorname{Hom}_{G}(Z G, A)=\{g: G \rightarrow A \mid g(x y)=x g(y), x, y \in G\} .
\end{aligned}
$$

Let $f \in A^{*}$ and assume $f+\operatorname{Im} \gamma \in \Gamma^{G}=\operatorname{Hom}_{G}(Z, \Gamma)$. Then $z f-f \in \operatorname{Im} \gamma$, $\forall z \in G$; so $(z f-f)(x y)=x(z f-f)(y)=x f(y z)-x f(y)$, and also $(z f-f)(x y)$ $=f(x y z)-f(x y), \quad \forall x, y, z \in G$. Let $z=y^{-1}$; then $f(x y)=x f(y)+f(x)-x f(1)$. Define $f_{c} \in \operatorname{Im} \gamma$ by $f_{c}(x)=x f(1)$; then $\left(f-f_{c}\right)+\operatorname{Im} \gamma=f+\operatorname{Im} \gamma$, and $f-f_{c} \in X(G, H, A)$. It is easily checked now that the map $f+\operatorname{Im} \gamma$ $\mapsto f-f_{c}$ is a natural isomorphism from $\operatorname{Hom}_{G}(Z, \Gamma)$ to $X(G, H, A)$.

Proposition 1.2. Let $H \subset G$ be groups, and let $A \in{ }_{G} \mathfrak{M}$. Then there exists a long exact sequence

$$
\begin{aligned}
0 \rightarrow A^{G} \stackrel{i}{\rightarrow} A^{H} \stackrel{\delta}{\rightarrow} H^{1}(G, H, A) \stackrel{j}{\rightarrow} H^{1}(G, A) & \stackrel{i}{\rightarrow} H^{1}(H, A) \stackrel{\delta}{\rightarrow} H^{2}(G, H, A) \stackrel{j}{\rightarrow} \ldots
\end{aligned}
$$

where the i's are restriction maps induced by the inclusion $H \rightarrow G$.

Proof. Apply $\operatorname{Ext}_{G}(Z,-)$ to the short exact sequence $0 \rightarrow A \rightarrow A^{*}$ $\rightarrow \Gamma \rightarrow 0 .\left(\operatorname{Ext}_{G}^{n}(Z, \Gamma(A)) \approx H^{n+1}(G, H, A)\right.$ by Lemma 1.1 , since $\Gamma(A)$ is exact and $\operatorname{Ext}_{G}(Z, \Gamma(A))$ is effaceable in ${ }_{G} \mathfrak{T}$.)

Corollaky 1.3. Let 1 denote the group with one element. Then $H^{n}(G, 1, A) \approx H^{n}(G, A), n \geqq 2, A \in{ }_{G} \mathfrak{T}$.

2. Let $H \subset G, L \subset K$ be groups. Let $\varphi: K \rightarrow G$ be a group homomorphism with $\varphi L \subset H$. If $A \in{ }_{G} \mathfrak{T}$, denote by $\Phi A$ the corresponding $K$-module structure in $A$ induced by $\varphi, x \cdot a=\varphi(x) a, x \in K, a \in A$. Then $\varphi$ induces a natural homomorphism $\varphi^{1}: X(G, H, A) \rightarrow X(K, L, \Phi A)$ defined by $\left(\varphi^{1} f\right) x=f(\varphi x)$, which in turn induces mappings $\varphi^{n}: H^{n}(G$, $H, A) \rightarrow H^{n}(K, L, A)$. If $\varphi$ is the inclusion we will denote $\Phi A$ by $A$ again.

Lemma 2.1. Let $H \subset K \subset G$ be groups. Then $\left\{H^{n}(K, H,-) \mid n \geqq 1\right\}$ is a universal sequence of connected functors in ${ }_{G} \mathrm{~T}$ (" $\partial$-foncteur universel" in the terminology of [6]).

Proof. The sequence is certainly exact. So, it suffices to show that it is effaceable (see [6, Proposition 2.2.1]). If $A$ is a $G$-injective module, then it is $K$-injective, since $Z G$ is $K$-free (see [4, p. 31, Proposition 6.2a]). Thus $H^{n}(K, H, A)=0$ if $n>1$.

Now let $H$ and $K$ be groups with a common subgroup $L$, and denote by $H *_{L} K$ the amalgamated product of $H$ and $K$ with amalgamated subgroup $L$ (i.e., the pushout of $L \rightarrow H$ and $L \rightarrow K$ in the category of groups), cf. [7, p. 312]. 
THEOREM 2.2 (EXCISION AxIOM). Let $L$ be a common subgroup of groups $H$ and $K$ and let $G=H *_{L} K$. Then the morphisms of functors in ${ }_{G} \mathfrak{T}$,

$$
\varphi^{n}: H^{n}(G, H,-) \rightarrow H^{n}(K, L,-), \quad n \geqq 1,
$$

induced by the inclusion $\varphi:(K, L) \rightarrow(G, H)$, are isomorphisms.

Proof. By Lemma 2.1, it suffices to show that $\varphi^{1}$ is an isomorphism. Let $A \in{ }_{G} \mathscr{M}$; if $f \in X(G, H, A)$ and $k \in K$, then, by definition, $\left(\varphi^{1} f\right) k=f k$. Consider the map $\psi: X(K, L, A) \rightarrow X(G, H, A)$ defined in the following manner. Let $g \in X(K, L, A)$ and $x \in G$; suppose $a_{1} a_{2}$ $\cdots a_{n}$ is a representative word of $x\left(a_{i}\right.$ belongs either to $H$ or to $K$, $i=1,2, \cdots, n)$. Then set

$$
(\psi g) x=g^{\prime}\left(a_{1}\right)+a_{1} g^{\prime}\left(a_{2}\right)+\cdots+a_{1} a_{2} \cdots a_{n-1} g^{\prime}\left(a_{n}\right)
$$

where $g^{\prime}\left(a_{i}\right)=g\left(a_{i}\right)$ if $a_{i} \in K$, and $g^{\prime}\left(a_{i}\right)=0$ if $a_{i} \in H$.

It is easily proved that $\psi \mathrm{g}$ is a well-defined crossed homomorphism of $G$ to $A$ vanishing on $H$, i.e. $\psi g \in X(G, H, A)$. Moreover, $\psi$ is a homomorphism.

On the other hand it is plain that $\varphi^{1} \psi=$ id. on $X(K, L, A)$; also, if $a_{1} a_{2} \cdots a_{n}$ is a representative word of $x \in G$, and $f \in X(G, H, A)$, then

$$
\begin{aligned}
\left(\psi \varphi^{1}\right)(f)(x) & =\left(\varphi^{1} f\right)^{\prime}\left(a_{1}\right)+a_{1}\left(\varphi^{1} f\right)^{\prime}\left(a_{2}\right)+\cdots+a_{1} a_{2} \cdots a_{n-1}\left(\varphi^{1} f\right)^{\prime}\left(a_{n}\right) \\
& =f\left(a_{1}\right)+a_{1} f\left(a_{2}\right)+\cdots+a_{1} a_{2} \cdots a_{n-1} f\left(a_{n}\right) \\
& =f\left(a_{1} a_{2} \cdots a_{n}\right)=f(x)
\end{aligned}
$$

i.e. $\psi \varphi^{1}=$ id. on $X(G, H, A)$. Thus $\varphi^{1}$ is an isomorphism.

Proposition 2.3. Let $G=H *_{L} K$ where $L$ is a common subgroup of groups $H$ and $K$. Then

$$
H^{n}(G, L, A) \approx H^{n}(H, L, A) \oplus H^{n}(K, L, A),
$$

for $n \geqq 1$ and $A \in{ }_{G} \mathfrak{M}$, where the canonical projections are induced by the inclusions $(H, L) \rightarrow(G, L)$ and $(K, L) \rightarrow(G, L)$.

Proof. By Lemma 2.1 it suffices to show that the result holds on dimension 1. If $f \in X(G, L, A)$, define $\varphi f=\left(f_{1}, f_{2}\right)$, where $f_{1} \in X(H, L, A)$ and $f_{2} \in X(K, L, A)$ are the restrictions of $f$ to $H$ and $K$ respectively. Conversely, given $g_{1} \in X(H, L, A)$ and $g_{2} \in X(K, L, A)$, define $\psi\left(g_{1}, g_{2}\right)=g: G \rightarrow A$ as follows: if $a_{1} a_{2} \cdots a_{n}$ is a representative word of $x \in G$, put $g(x)=g^{\prime}\left(a_{1}\right)+a_{1} g^{\prime}\left(a_{2}\right)+\cdots+a_{1} a_{2} \cdots a_{n-1} g^{\prime}\left(a_{n}\right)$, where $g^{\prime}\left(a_{i}\right)$ is $g_{1}\left(a_{i}\right)$ or $g_{2}\left(a_{i}\right)$ depending on whether $a_{i}$ is in $H$ or $K$ respectively (notice that $g_{1 \mid L}=g_{2 \mid L}=0$ ). Then $g$ is a well-defined crossed 
homomorphism of $G$ to $A$ vanishing on $L$, i.e. $g \in X(G, L, A)$. Hence $\varphi$ and $\psi$ are inverse isomorphisms as desired.

REMARK. Proposition 2.3 has also been proved independently by M. Barr and J. Beck; see [2].

Corollary 2.4 (Lyndon [8]; Barr And Rinehart [3]). Let $G=H * K$ (free product of groups $H$ and $K$ ) and let $A \in{ }_{G} \mathrm{~N}$. Then $H^{n}(G, A)=H^{n}(H, A) \oplus H^{n}(K, A)$ if $n \geqq 2$.

Proof. Put $L=1$ in Proposition 2.3 and apply Corollary 1.3.

We now prove a converse to Proposition 2.3. Notice first that given a group $T$ and an abelian group $B$, a $T$-module structure on $B$ is nothing but a group homomorphism $T \rightarrow \operatorname{Aut}(B)$, where $\operatorname{Aut}(B)$ is the group of automorphisms of $B$.

Proposition 2.5. Let $H$ and $K$ be subgroups of a group $G$, and let $L=H \cap K$. Assume that for every abelian group $A$ and every pair $\varphi_{1}: H \rightarrow \operatorname{Aut}(A), \varphi_{2}: K \rightarrow \operatorname{Aut}(A)$ of group homomorphisms that coincide on $L$ there is a group homomorphism $\varphi: G \rightarrow \operatorname{Aut}(A)$ extending $\varphi_{1}$ and $\varphi_{2}$. Suppose, moreover, that the isomorphisms of Proposition 2.3 hold. Then $G=H *_{L} K$.

Proof. In particular

$$
X(G, L, A) \approx X(H, L, A) \oplus X(K, L, A)
$$

for $A \in{ }_{G} \mathfrak{T}$, i.e. every pair $f_{1}: H \rightarrow A, f_{2}: K \rightarrow A$ of crossed homomorphisms vanishing on $L$ extends uniquely to a crossed homomorphism $f: G \rightarrow A$. Let $G_{1}$ be the subgroup of $G$ generated by $H$ and $K$; we first show that $G=G_{1}$. Assume $G \neq G_{1}$. If $x \in G$, let $\bar{x}$ denote the corresponding left coset of $G_{1}$ in $G$. Let $I=I\left(G / G_{1}\right)$ be the free abelian group generated by $\{\bar{x}-1 \mid 1 \neq x \in G\}$, and let $G$ act on $I$ by $y(\bar{x}-1)$ $=\left((y x)^{-}-1\right)-(\bar{y}-1), x, y \in G$. Then $I \in{ }_{G} \Re$. Let $f_{1}: H \rightarrow I$ and $f_{2}: K \rightarrow I$ be the zero crossed homomorphisms; these extend to the zero crossed homomorphism $G \rightarrow I$. On the other hand $f: G \rightarrow I$, defined by $f x=\bar{x}-1, x \in G$, is plainly a nonzero crossed homomorphism extending $f_{1}$ and $f_{2}$, contradicting the hypothesis. Hence $G=G_{1}$.

We will see now that

$$
\begin{gathered}
L \rightarrow H \\
\downarrow \\
\quad \downarrow \\
K \rightarrow G
\end{gathered}
$$

is a pushout diagram (all maps are inclusions), i.e. $G=H *_{L} K$. Suppose $P$ is a group and let $\varphi_{1}: H \rightarrow P$ and $\varphi_{2}: K \rightarrow P$ be group homomor- 
phisms that coincide on $L$. Denote by $F(P)$ the free abelian group on the set $P$, and consider a standard embedding $P \rightarrow \operatorname{Aut}(F(P))$. Then by assumption $\varphi_{1}$ and $\varphi_{2}$ extend to a group homomorphism $\varphi$ : $G$ $\rightarrow \operatorname{Aut}(F(P))$. However, since $G$ is generated by $H$ and $K, \varphi$ must be unique and into $P$.

Finally, we state the following proposition whose proof is formally as in Theorem 15.3(c), p. 43 of [5], and which is therefore omitted.

Proposition 2.6 (A Mayer-Vietoris Sequence). Let $L, H, K, G$ and $A$ be as in Theorem 2.2. Then the sequence

$$
\begin{aligned}
\ldots \rightarrow H^{q-1}(L, A) \stackrel{\Delta}{\rightarrow} & H^{q}(G, A) \\
& \stackrel{\phi}{\rightarrow} H^{q}(H, A) \oplus H^{q}(K, A) \stackrel{\Psi}{\rightarrow} H^{q}(L, A) \rightarrow \ldots
\end{aligned}
$$

where $\Delta=H^{q-1}(L, A) \stackrel{\delta}{\rightarrow} H^{q}(K, L, A) \stackrel{\left(\varphi^{q}\right)^{-1}}{\rightarrow} H^{q}(G, H, A) \rightarrow H^{q}(G, A)$ with $\delta$ and $j$ as in Proposition 1.2, and $\varphi^{q}$ as in Theorem $2.2 ; \phi$ is the direct sum of the maps induced in cohomology by the inclusions $H \rightarrow G$ and $K \rightarrow G$; $\Psi\left(v_{1}, v_{2}\right)=h_{1}^{q} v_{1}-h_{2}^{q} v_{2}$, where $h_{1}^{q}$ and $h_{2}^{q}$ are maps induced in cohomology by the inclusions $h_{1}: L \rightarrow H$ and $h_{2}: L \rightarrow K$ respectively, $v_{1} \in H^{a}(H, A)$, $v_{2} \in H^{q}(K, A)$.

\section{REFERENCES}

1. M. Auslander, Thesis, Columbia University, 1954.

2. M. Barr and J. Beck, Homology and standard constructions. Seminar on triples and categorical homology, Lectures Notes, Springer-Verlag, New York, 1968.

3. M. Barr and G. S. Rinehart, Cohomology as the derived functor of derivations, Trans. Amer. Math. Soc. 122 (1966), 416-426.

4. H. Cartan and S. Eilenberg, Homological algebra, Princeton Univ. Press, Princeton, N. J., 1956.

5. S. Eilenberg and N. Steenrod, Foundations of algebraic topology, Princeton Univ. Press, Princeton, N. J., 1952.

6. A. Grothendieck, Sur quelques points d'algèbre homologique, Tôhoku Math. J. 9 (1957), 119-221.

7. M. Hall, The theory of groups, Macmillan, New York, 1959.

8. R. C. Lyndon, Cohomology theory of groups with a single defining relation, Ann. of Math. (2) 52 (1950), 650-665.

UNIVERSITY OF ILLINOIS 